\title{
The SYBAR system: Integrated recording and display of video, EMG, and force plate data
}

\author{
JAAP HARLAAR, RONALD A. REDMEIJER, \\ PETER TUMP, ROB PETERS, and EDWIN HAUTUS \\ Free University, Amsterdam, The Netherlands
}

\begin{abstract}
A new system - called SYBAR-is introduced, that employs digital video for registration of the movements of a patient while simultaneously recording electromyogram signals of relevant muscles and ground reaction forces (for the lower extremities in gait studies). All information is stored in a multimedia record, which can be viewed by the clinician with a simple user interface. This setup allows an integrated and more detailed view of the movement of the patient and related information (i.e., muscle physiology). It is used by clinicians to assess the causes of movement disorders in their patients. This paper describes the SYBAR system and focuses on the employed methods of data synchronization for both the time and the spatial domains. It is concluded that, although SYBAR was developed for clinical gait studies, the technology can be applied in all situations in which the relation between physiological signals and human or animal behavior is studied.
\end{abstract}

Movement disorders might imply that the patient has a functional disability. In such a case, the patient is not able to perform the activities of daily living at the level at which he or she aims. Therefore, in rehabilitation medicine, the assessment of motor dysfunction is an important part of the diagnosis for the indication of therapy. Such assessment includes an analysis of motor disorders through careful observation of the patient, who demonstrates his disability on a test of motor performance (e.g., walking). A phenomenological description of observed gait disorders is common in regular clinical practice, and reporting is performed accordingly. Although human movement is enormously complex, it seems that physiatrists, orthopedic surgeons, physical therapists, and so forth share an implicit comprehension of it and are well able to recognize specific deviations of human movement. Moreover, the physician tries to infer possible impairments of, for example, joint and muscle functions that may be causing an experienced disability (Harlaar \& Lankhorst, 1998). Although observation of the movement is the first choice for a clinician in the assessment of movement disorders, careful analysis by the unaided senses does not unambiguously bring to light the causal factors for these disorders. An implicit musculoskeletal model is used to analyze human movement, and an observer translates the posture and the movement of the subject into contractions of the muscles and into the imposed load of the joint. However, muscle function has an indeterminate relation to posture and movement (i.e., there are more muscles than degrees

Danny Koops and Cor Klok are acknowledged for their assistance in engineering the calibration frame. Correspondence concerning this article should be addressed to J. Harlaar, University Hospital, Vrije Universiteit, De Boelelaan 1117, P.O. Box 7057, 1007 MB Amsterdam, The Netherlands (e-mail: j.harlaar@azvu.nl). of freedom of the human skeleton), resulting in an infinite number of possible combinations of muscular contractions. Therefore, additional information is necessary, which can be obtained by measuring muscle activity by means of the surface electromyogram (EMG). A second problem for the clinician is to infer joint load from the observation. Principally, the human skeleton can be considered as a mechanical system for which the kinematics, together with the posture in a gravitational field, are uniquely related to the kinetics of the system by Newtonian laws (i.e., inverse dynamics). However, inasmuch as such an analysis for the joints of the lower extremities does not follow easily from observation in, for example, gait studies, the measurement of the ground reaction force (GRF) is very helpful.

In order to relate a video of the movement, the EMG, and the GRF to each other in a relevant way, it is first necessary to record these signals in a time-synchronized manner. For the GRF, it is also necessary to apply spatial synchronization. Finally, all these signals must be presented in a meaningful way to a physician who is familiar with observational analysis. To do so, we developed SYBAR, which is a Dutch acronym for system for movement analysis in a context of rehabilitation medicine (Harlaar \& Hautus, 1995; Hautus, 1997). SYBAR is based on multimedia technology that integrates digitized video and digitized (physiological) analog signals.

\section{INSTRUMENTATION}

\section{Introduction}

Integrated analysis of human movement and physiological signals in a clinical context consists basically of three parts: recording, in which the signals are recorded in a time-synchronized manner; processing, which builds the multimedia records by which to establish time and 


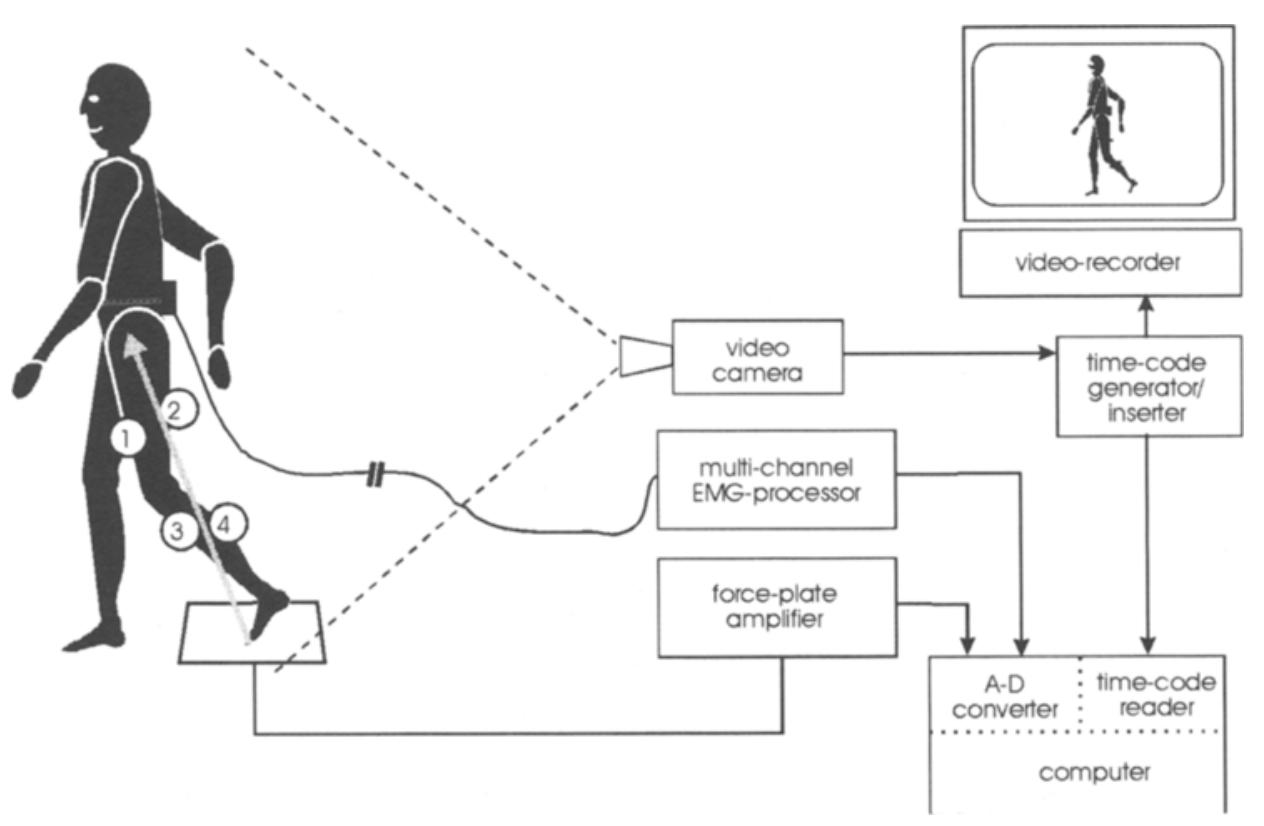

Figure 1. Overview of the instrumentation setup during recording.

space synchronization; and viewing, which is an interactive display of the multimedia records in a way that is meaningful to the user (i.e., the physician).

\section{Instrumentation for Recording}

The three types of recording that were performed in a common examination of the gait pattern of a patient are video, kinesiological EMG, and GRF (Figure 1). A walkway of $11.5 \times 2 \mathrm{~m}$ was constructed, from which the central $5.5 \mathrm{~m}$ were used to record the gait. Also, other human activities of daily living were studied in this way (e.g., rising from a chair or jumping).

Video. Video recording is used for registration of the movement, to serve observational analysis. We used a custom CCD video camera with an electronic shutter set at $1 / 125 \mathrm{sec}$, to prevent movement fuzziness. Two types of scenes for registration of the patient were distinguished. For frontal recording of walking and for recording from a stationary point of view, a camera on a tripod was used (e.g., for rising from a chair or for activities related to the upper extremity). For recording of walking in the sagittal plane, a camera was used that could be moved along a rail that was mounted close under the ceiling, parallel to the actual walkway. In this way, several strides could be recorded, using a constant perspective. However, for recordings that involved measuring the GRF, a stationary camera was used. Besides a single view from one camera, a screen splitter was used to combine the images of two cameras to create a double view of a single scene (e.g., the frontal and sagittal view of a gait).

Kinesiological electromyogram. For the leadoff of kinesiological EMG, we used surface electromyography, which is appropriate for addressing the larger superficial muscles of the lower extremities. A bipolar measuring technique ensured a muscle-specific signal leadoff that, in combination with the proper instrumentation, provided a reliable signal (Hof, 1984). A pair of disposable electrodes (Meditrace pellet electrodes) were used with a 23-mm rim-to-rim distance between the pair of electrodes. The surface area of each electrode measured 10 $\times 10 \mathrm{~mm}$. The EMG signal was preamplified, with a miniature amplifier (gain, $100 \times$; CMRR, $>100 \mathrm{~dB}$; input impedance, $>100 \mathrm{M} \Omega$; RMS noise, $<1.0 \mu \mathrm{V})$ di-
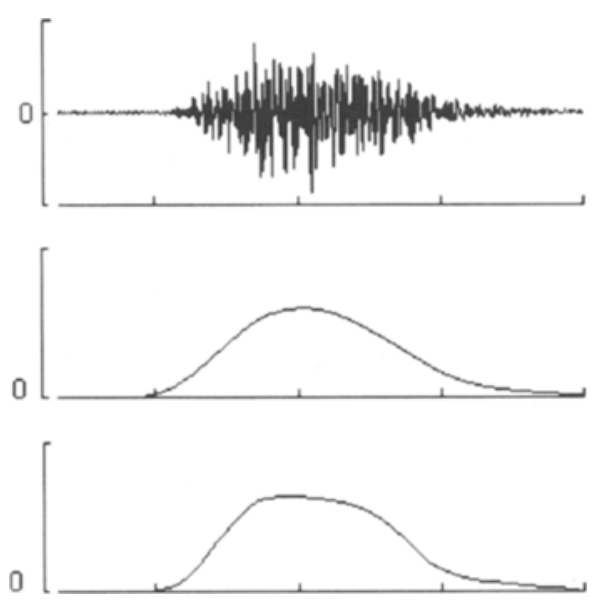

Figure 2. Relation between electromyogram (EMG), smoothed rectified EMG, and muscular force. Upper trace, surface EMG of the $\mathrm{m}$. vastus lateralis; middle trace, smoothed rectified EMG; lower trace, the net moment of force about the knee; abcissa, time, with 1-sec marks; ordinates, arbitrary units. 


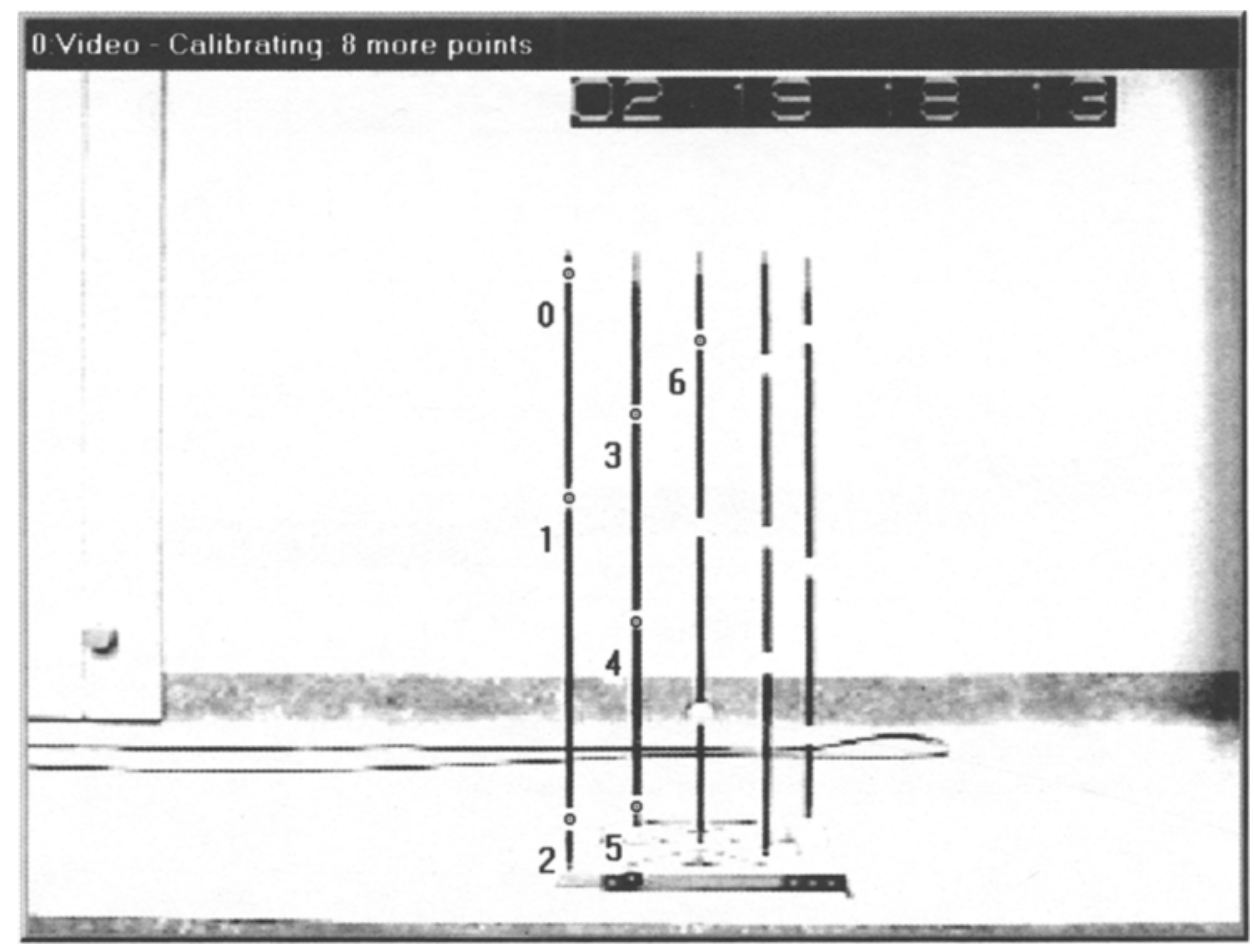

Figure 3. Calibration frame on the force plate. The first seven markers are indicated by the operator.

rectly mounted on the pair of electrodes. This resulted in a signal that was no longer vulnerable to distortion and could be led away from the body through a 10 -m-long "umbilical" that conveyed all the channets to the EMG processor. Here, the EMG signal was high-pass filtered (cutoff frequency, $20 \mathrm{~Hz}, 18 \mathrm{db} / \mathrm{oct}$ ) in order to suppress any residual movement artifacts and was low-pass filtered at $1500 \mathrm{~Hz}$ to limit bandwidth. Final gain was controlled by the operator, who carefully inspected the EMG signal on a paper chart recorder during a first trial, aiming at maximal amplification but avoiding saturation. This yielded the so-called "raw" EMG signal, being a noisy signal (Figure 2). Subsequent signal processing involved rectification and low-pass filtering, resulting in the smoothed rectified EMG (SR-EMG). For our application, we used a second-order low-pass filter with the cutoff frequency set at $2.0 \mathrm{~Hz}$ so that the dynamics of the SR-EMG roughly approximated those of the force (Figure 2; Calvert \& Chapman, 1977; Crosby, 1978; Olney \& Winter, 1985). An eight-channel EMG system was used.

Ground reaction force. The GRF was measured with a force plate that was immersed in the walkway. Three orthonormal components of both force and moment were recorded. All six signals were low-pass filtered at $5 \mathrm{~Hz}$. To transform the recorded signals into coordinates of the video image, each time a camera position was changed, an additional video recording of a calibration frame was made (Figure 3 ). This frame was put on top of the force plate and comprised 15 clear markers. These markers had well-known coordinates and were evenly distributed over the space above the force plate $(40 \times 40 \times 100 \mathrm{~cm})$.

Data acquisition. During the video recording of the subject, which was stored on videotape, a time code was generated at the trigger of each new frame of the video signal (time-code generator, TC30 by Alpermann + Velte GmbH, Remscheid, Germany). This time code was included in the video signal (and thus stored on tape) as a so-called vertical interval time code (VITC; see Figure 4). The time-code generator also inserted the time code as readable numbers in the video image. All analog signals were sampled at a frequency of $100 \mathrm{~Hz}$ and were digitized with a PC-based analog-to-digital converter. Simultaneously with each sample, the actual VITC of the video frame was read out from the video signal. All the samples (containing the VITC and the actual value of each of the SR-EMG and force plate channels for each time instance) were stored in a computer file.

\section{Instrumentation: Processing}

After recording is done as described above, the video recording of the activity is digitized, using a video digitizer (Miro DC30 by Miro Computer Products A.G., Braunschweig, Germany). During this off-line process, the appropriate part of the video recording is selected and digitized. This results in a so called AVI video file, which is played back in a separate video window. Then the operator adds the time code of the first video frame to the system setup, before importing the measurement files, which comprise subsequent time samples of synchronous 

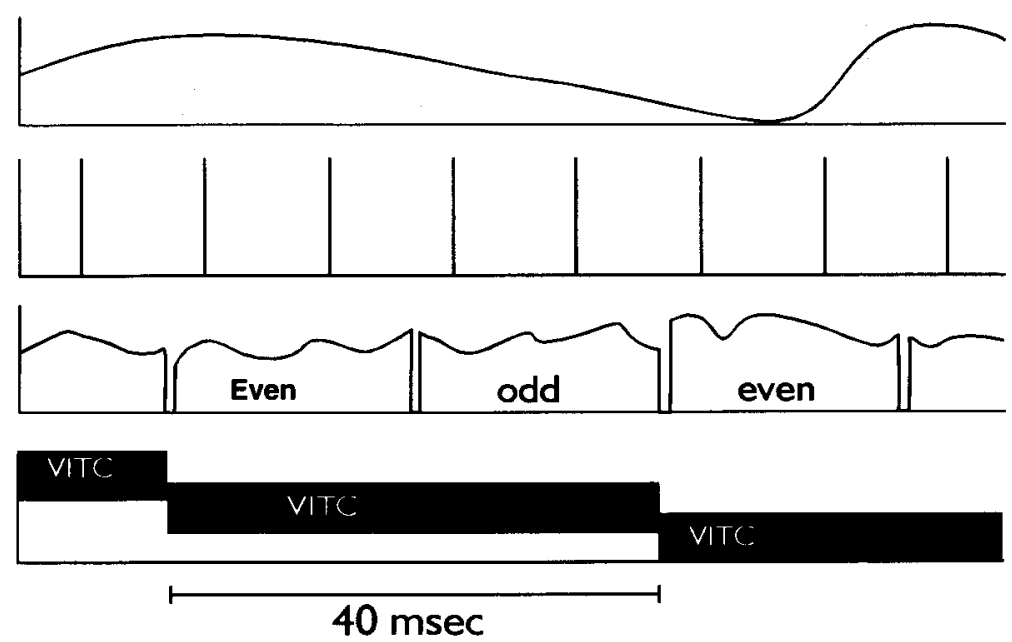

Figure 4. Synchronization of signals. From top to bottom: the analog signal; the trigger strobes (at $100 \mathrm{~Hz}$ ) used for analog-to-digital conversion; the (simplified) video signal, clearly showing the Vsync pulses (odd and even fields are indicated); the actual generated time code ( $n=$ arbitrary frame number).

VITC, SR-EMG, and force values. When several samples of signal values are annotated with the same VITC, these samples are averaged. In our case, signals were sampled at $100 \mathrm{~Hz}$, and the video frame rate was $25 \mathrm{~Hz}$, resulting in the averaging of four signal samples to obtain a single value per video image. Separate windows are created to display the analog signals as time-dependent graphs. SREMG is also displayed as dynamic bar graphs. In the case of force plate measurements, an image of the calibration frame is also digitized (Figure 3 ). The operator identifies the markers of the frame on the screen by mouse clicking. Using the a priori real-world coordinates and screen coordinates of the markers, transformation parameters are calculated on the basis of the direct linear transformation algorithm (Wood \& Marshall, 1986). From the measured components of force and moment, the origin magnitude and position of the ground reaction vector is calculated for each video frame. The transformation parameters are used to calculate where the GRF vector should be drawn in the video window as an overlay to the actual image. This so-called multimedia record is stored for off-line retrieval by a physician for interpretation.

\section{Instrumentation: Viewing}

In Figure 5, a screen print is shown that illustrates how the multimedia records are presented to the end user of the SYBAR system. Playback of the video is controlled with a familiar VCR user interface (i.e., the "play," "stop," "home," "end," and "frame forward/backward" buttons). Moreover, to hide the EMG, signals can be toggled to hidden versus present by clicking the bar graphs of the specific EMG signal, named after the muscle. The actual time instant of the video image in the time-based graphs is indicated by a hairline cursor. Clicking a specific point in the time-based graph will immediately synchronize the corresponding video image. In this way, the clinician can analyze the movement of the patient interactively. Movement analysis by careful observation serves the identification of possible causes at the level of muscle impairment to assist clinical decision making (Harlaar \& Lankhorst, 1998). Adding explicit measures (i.e., the activation levels of the muscles, as well as the projection of the GRF onto the body) provides information for a better estimation of these functions. In this way, clinical decision making for optimal treatment is served.

\section{DISCUSSION}

\section{EMG}

The application of surface electromyography during movement (i.e., kinesiological EMG) is limited to the larger superficial muscles. The time-dependent intensity of the EMG signal is considered to be a meaningful property of kinesiological EMG for the study of muscle coordination. There are several methods by which to quantify this property, but the most appealing and viable way is to use the linear envelope (Winter, 1984). The linear envelope is processed as the SR-EMG and is nowadays the de facto standard in processing EMG for kinesiological applications. The choice of $2.0 \mathrm{~Hz}$ is also well below the sample frequency of the movement, being the video frame rate $(25 \mathrm{~Hz}$ in Europe).

\section{Ground Reaction Force}

To estimate the net moment of force about lower extremity joints in studies of posture and gait, the GRF as well as the position of the joint center of rotation, must be measured. Not only in formal calculation, but also in observational analysis, the projection of the GRF vector onto the body is an effective aid in the estimation of the 


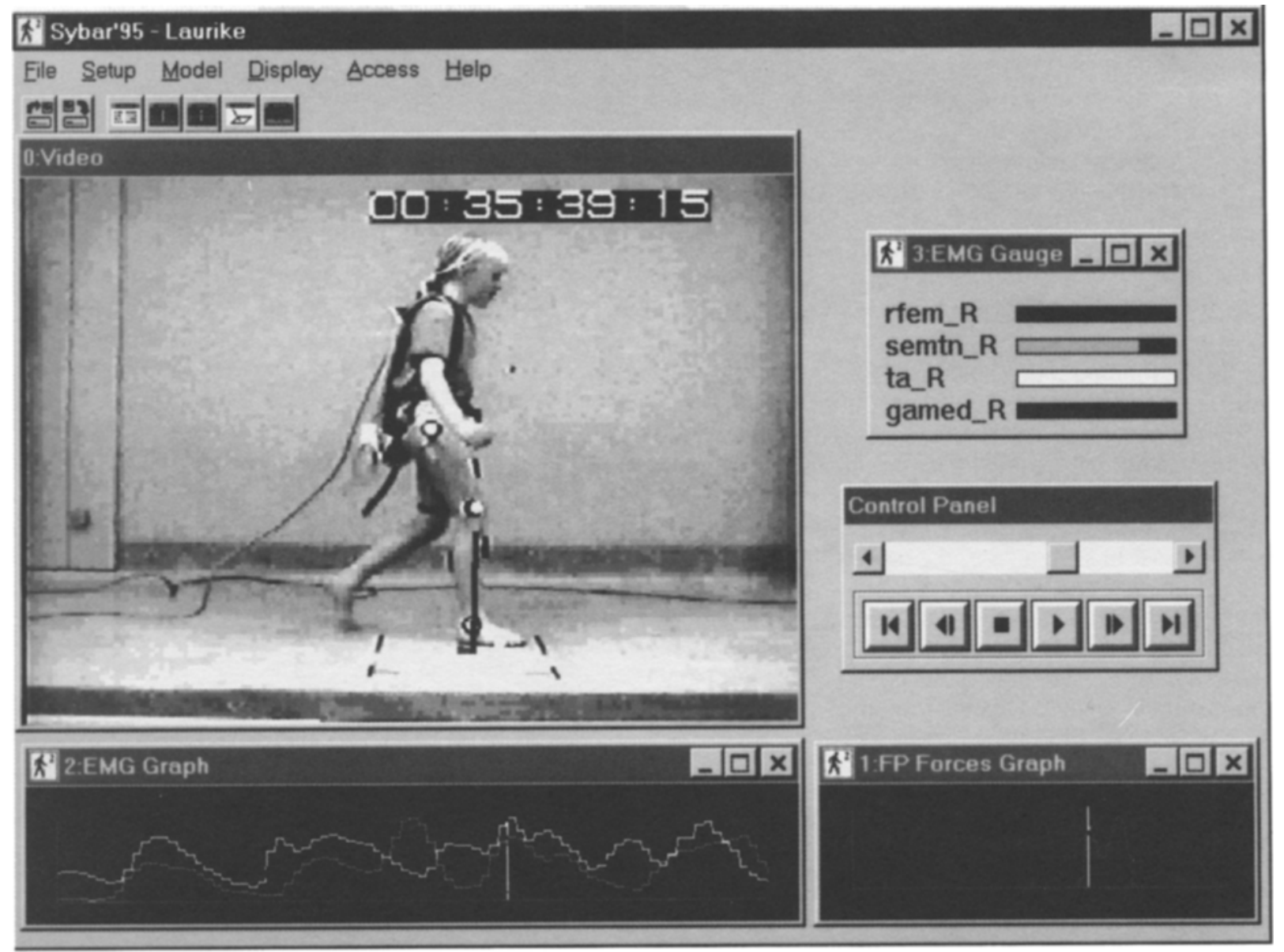

Figure 5. Typical screen of SYBAR used as a viewer.

net moment of force around a joint (Boccardi, Pedotti, Rodano, \& Santambrogio, 1981; Lanshammer, 1988). In quantitative gait analysis, the correct net moments of force are calculated by using additional inverse dynamics of segments distal of the joint (see, e.g., Davis, Ounpuu, Tyburski, \& Gage, 1991; Kingma, de Looze, Toussaint, Klijnsma, \& Bruijnen, 1996). To do so, accurate kinematics, as measured by a motion capture system, is necessary. However, it was shown that the increase in accuracy from using a complete inverse dynamics approach is limited in normal gait (Boccardi et al., 1981; Lanshammer, 1988). Therefore, the accuracy of an estimation of net joint load from using the projection of the GRF (dynamic plumb line) seems appropriate as a first-order approximation.

\section{Multimedia Technology}

Previous realizations of the simultaneous display of EMG and movement through the use of a split-screen technique, the second camera viewing the instrument's display (Winter, 1984), have been reported. A GRF video overlay was reported in which a half-silvered mirror between scene and oscilloscope was used (Boccardi et al., 1981). Less cumbersome solutions were achieved by em- ploying an analog electronic video overlay, in combination with a computer for data acquisition and signal processing, for both GRF (Lanshammer, 1988) and EMG (Harlaar, 1991). However, these solutions still use a videocassette for recording and playback. As was argued above, the use of digital video enhances accessibility of patient records, as well as efficient interactive analysis. Owing to the fixed frame rate, the use of video might be too limited for fast movements (e.g., jumping, kicking, or running).

\section{Synchronization}

In the present implementation of time synchronization, the industry standard for video frame identification is used - that is, the VITC. However, as can be concluded from Figure 4, this code does not selectively code the even and odd fields that span up a single video frame. More professional versions of VITC generators might be able to code a difference between odd and even frames. However, in such a case too, the digitizing soft- and hardware must also be able to capture either even or odd fields selectively, to establish a closer synchronicity. Since information technology is rapidly progressing, these developments are expected in due course. The solution of two 
other problems would increase the robustness of time synchronization. First, unfortunately, the time code is not embedded in the AVI file format and must, therefore, be added by the SYBAR-system. On the basis of the first image that is captured, the inserted time code in the image (which is human readable), is added to the setup. This procedure makes the synchronization vulnerable to occasional dropouts of fields during the digitization process. The most robust solution to this problem is to use a video format in which the time code is linked to each video frame. Second, the clocks that trigger the video signal and the analog-to-digital conversion of the analog signals are running independently. This problem is currently dealt with by capturing the analog signals at a rate of $100 \mathrm{~Hz}$, creating a higher time resolution than does the video signal. This could easily be solved by using the Vsync of the video signal as a master and triggering the analog-to-digital conversion and the VITC readout as a slave process. In such a case, the design should operate with minimal time delay in the system. A hardwareembedded system, in which the triggering and capturing both the analog signals (through analog-to-digital conversion) and the VITC are integrated, is expected to provide a closer synchronization than does software running in a multitasking operation system.

\section{Generalization}

The SYBAR multimedia system is used for the analysis of muscle function, muscle coordination, and joint load. The combination of (nonobservable) physiological signals and digital video is used to assist the analysis of the disabled activities of a patient in terms of impairments of muscle and joint function. This application is linked to the biomechanical analysis of movements, rather than to the measurement and analysis of human or animal behavior. However, the same system can be used to integrate physiological signals and digitized videos of human or animal behavior. Any physiological signal can be included in the system, as long as its bandwidth is compatible with the capturing rate of video images. For most physiological signals or the parameters derived from them (e.g., heart rate, respiration rate, blood pressure, EEG), this is the case. With respect to the integrated analysis of behavior $T$ and physiological signals, the above-mentioned limitation in time accuracy (of about one frame) seems less of a problem than it is in motion analysis. In conclusion, the use of multimedia technol- ogy enables the visualization of physiological functions in a way that is meaningful to the researcher - that is, in a way that directly fits current concepts of physiology. The strength of such visualization is that it will assist the researcher in hypothesizing relations between behavior and physiology, prior to formal quantitative evaluation.

\section{REFERENCES}

Boccardi, S., Pedotti, A., Rodano, R., \& Santambrogio, G. C. (1981). Evaluation of muscular moments at the lower limb joints by an on-line processing of kinematic data and ground reaction. Journal of Biomechanics, 14, 35-45.

Calvert, T. W., \& Chapman, A. E. (1977). The relationship between the surface EMG and force transients in muscle: Simulation and experimental studies. Proceedings of the IEEE, 65, 682-689.

Crosiy, P. A. (1978). Use of surface electromyogram as a measure of dynamic force in human limb muscles. Medical \& Biological Engineering \& Computing, 16, 519-524.

Davis, R. B., Ounpuu, S., Tyburski, D., \& Gage, J. R. (1991). A gait analysis data collection and reduction technique. Human Movement Science, 10, 575-587.

HARLAAR, J. (1991). The clinical use of biomechanics in gait analysis. Journal of Rehabilitation Sciences, 4, 35-40.

HarlaAR, J., \& Hautus, E. H. (1995). Multi-media technology for movement analysis to assist clinical decision making [Abstract]. In K. Häkkinen, K. L. Keskinen, P. V. Komi, \& A. Mero (Eds.), Proceedings of the XVth Congress of the International Society of Biomechanics (p. 362). Jyväskylä, Finland: University of Jyväskylä.

HARLAAR, J., \& LANKHORST, G. J. (1998). Impairment assessment in rehabilitation medicine: The potential role of technology to assist clinical decision making. Disability \& Rehabilitation, 20, 29-32.

HAUTUS, E. H. (1997). SYBAR, a human motion analysis system for rehabilitation medicine. Unpublished doctoral dissertation, Technische Universiteit Eindhoven, Eindhoven, The Netherlands.

HoF, A. L. (1984). EMG and muscle force: An introduction. Human Movement Science, 3, 119-153.

Kingma, I., de Looze, M. P., Toussaint, H. M., Klijnsma, H. G., \& Bruijnen, T. B. M. (1996). Validation of a full-body 3-D dynamic linked segment model. Human Movement Science, 15, 833-860.

LANSHAMmER, H. (1988). VIFOR: A system for force line visualization. In A. P. Hollander, G. de Groot, P. A. Huijing, \& G. J. van Ingen Schenau (Eds.), Biomechanics $X I-B$ (p. 984). Amsterdam: Free University Press.

Olney, S. J., \& Winter, D. A. (1985). Predictions of knee and ankle moments of force in walking from EMG and kinematic data. Journal of Biomechanics, 18, 9-20.

Winter, D. A. (1984). Pathological gait diagnosis with computeraveraged electromyographic profiles. Archives of Physical Medicine \& Rehabilitation, 65, 393-398.

WoOD, G. A., \& MARSHALL, R. N. (1986). The accuracy of DLT extrapolation in three-dimensional film analysis. Journal of Biomechanics, $19,781-785$.

(Manuscript received December 18, 1998; revision accepted for publication November 2, 1999.) 